SOI: 1.1 /TAS DOI: $10.15863 /$ TAS

International Scientific Journal Theoretical \& Applied Science

p-ISSN: 2308-4944 (print) $\quad$ e-ISSN: 2409-0085 (online)

Year: $2016 \quad$ Issue: 1 Volume: 33

Published: $30.01 .2016 \quad$ http://T-Science.org

SECTION 20. Medicine.
Marya Petrovna Salekhova $\mathrm{PhD}$, cardiologist "Alanda clinic "Kazakhstan salekhova_m@mail.ru

Gaukhar Tleubekkyzy Igimbayeva $\mathrm{PhD}$, Republic coordinator CPDHW CSD,

Assos. Professor, Kazakhstan igt_tgt@mail.ru

Said Abdullaevich Salekhov Professor, doctor of medical sciences Novgorod State University of Yaroslav Mudry, Russia ssalehov@mail.ru

Timur Kenzheshovich Utegaliev Heart surgeon,Cardiac Surgery Center Mangistau regional hospital, Kazakhstan utk.75@mail.ru

Aivar Melsovich Kenzhebaev Deputy Director of the City Heart Center Almaty, Kazakhstan iwtk@mail.ru

\title{
PATHOGENETIC SUBSTANTIATION OF CORRECTION OF FUNCTIONAL INTESTINAL DISORDERS IN POSTERIOR MYOCARDIAL INFARCTION
}

\begin{abstract}
We were analyzed peculiarities of post-MI period in 76 patients with posterior myocardial infarction, depending on the use of targeted prevention and correction of violations of the functional state of the intestine. Patients were divided into 2 groups. Group I included 34 patients who had post-MI prevention of violations of the functional state of the digestive tract caused by the cardiac-enteric inhibitory reflex was not carried out. In group II, 42 patients were activities aimed at the normalization of the functional state of the intestine and correction of consequences of cardiac-enteral inhibitory reflex in post-MI period. To do this, if necessary, we performed decompression of the stomach, holding permanent blockades retroperitoneal nerve plexus and the appointment Forlaks 1 standard package, diluted with $150 \mathrm{~mL}$ of water 6 times a day. We have found that when carrying out a complex of measures aimed at the normalization of the functional state of the intestine in patients with transmural posterior myocardial infarction was noted as a significant decrease in the frequency of intestinal paresis, and, a significant reduction of cardiac arrhythmias. This indicated pathogenetic feasibility of implementing the prevention of cardiac-enteral and enteral-cardiac reflexes.

Key words: posterior myocardial infarction, cardial enteral reflex, enteral cardial reflex, enteroparesis, heart rhythm disorder, permanent blockade, retroperitoneal nervous interlacement.

Language: Russian

Citation: Salekhova MP, Igimbayeva GT, Salekhov SA, Utegaliev TK, Kenzhebaev AM (2016) PATHOGENETIC SUBSTANTIATION OF CORRECTION OF FUNCTIONAL INTESTINAL DISORDERS IN POSTERIOR MYOCARDIAL INFARCTION. ISJ Theoretical \& Applied Science, 01 (33): 184-189.

Soi: http://s-o-i.org/1.1/TAS-01-33-33 Doi: crossef http://dx.doi.org/10.15863/TAS.2016.01.33.33
\end{abstract}

\section{ПАТОГЕНЕТИЧЕСКОЕ ОБОСНОВАНИЕ КОРРЕКЦИИ ФУНКЦИОНАЛЬНЫХ НАРУШЕНИЙ КИШЕЧНИКА ПРИ ИНФАРКТЕ ЗАДНЕЙ СТЕНКИ МИОКАРДА}

Аннотация: Были проанализированы особенности течения постинфарктного периода у 76 больных с инфарктом задней стенки миокарда в зависимости от применения целенаправленной профилактики и коррекции нарушений функционального состояния кишечника. Больных разделили на 2 группь. В I группу вошли 34 больных, которым в постинфарктном периоде профилактика нарушений функционального состояния пищеварительного тракта, обусловленных кардиально-энтеральным тормозным рефлексом, не проводилась. Во II у 42 больных в постинфарктном периоде проводились мероприятия, направленные на 
нормализацию функционального состояния кишечника и коррекциию последствий реализации кардиальноэнтерального тормозного рефлекса. Для этого при необходимости проводили декомпрессию желудка, проведение перманентных блокад забрюшинных нервных сплетений и назначение форлакса по 1 стандартному пакету, разведенному на 150 мл воды 6 раз в сутки. Было установлено, что при проведении комплекса мероприятий, направленных на нормализацию функиионального состояния кишечника у больных с трансмуральным инфарктом задней стенки миокарда отмечалось как достоверное уменьшение частоты парезов кишечника, так и, достоверное снижение нарушений сердечного ритма, что свидетельствовало о патогенетической целесообразности профилактики реализации кардиальноэнтерального и энтерально-кардиального рефлексов.

Ключевые слова: инфаркт задней стенки миокарда, кардиально-энтеральный рефлекс, энтеральнокардиальный рефлекс, парез кишечника, нарушение сердечного ритма, перманентная блокада, забрюшинные нервные сплетения.

Высокая распространенность сердечнососудистых заболеваний, сопровождающиеся высокой летальностью, тенденция к развитию и манифестации в более молодом возрасте [1, 2, 3, 4], позволяют отнести их к «болезням образа жизни» или иначе «болезням цивилизации» [5].

Особого внимания заслуживает атипичное течение инфаркта миокарда, в частности его абдоминальная форма, которая встречается лишь в $1,8-2,5 \%$ случаев и сопровождается клинической симптоматикой острых заболеваний органов брюшной полости. При абдоминальной форме инфаркта миокарда наиболее часто происходит трансмуральное поражение задней, либо нижне-диафрагмальной стенки миокарда [6].

Следует отметить, что болевой синдром может быть следствием как абдоминальной патологии, так и за счет иррадиации при патологии других органов, в том числе и сердца [7] дифференциальная диагностика между кардиогенными болями и острыми заболеваниями брюшной полости весьма затруднительна [8; 9]. При этом абдоминальный болевой синдром и сопутствующая ему симптоматика (тошнота, рвота, вздутие живота, задержка отхождения стула и газов, увеличенный и определяемый при пальпации желчный пузырь) свидетельствуют о функциональных нарушениях органов брюшной полости, что обусловлено реализацией разновидностей патологического кардиально-абдоминального тормозного рефлекса, в том числе кардиально-энтерального [10], кардиально-илеоцекального [11], кардиально-анального [12].

Учитывая, что на фоне ишемии миокарда при абдоминальной форме инфаркта миокарда появляются функциональные нарушения органов брюшной полости, было высказано предположение, что при инфаркте миокарда без манифестации клинической симптоматики со стороны органов брюшной полости все равно будут развиваться нарушения их функционального состояния, что может иметь значение для течения постинфарктного периода

\section{Особого} внимания заслуживает функциональное состояние кишечника, угнетение перистальтики которого в постинфарктном периоде, может привести к развитию пареза, либо, при осложненном течении к функциональной непроходимости кишечника [1].

ЦЕЛЬ: Обосновать патогенетическую целесообразность проведения профилактики и лечения нарушений моторной и эвакуаторной функции кишечника при инфаркте задней стенки миокарда.

\section{Материалы и методы}

Были изучены особенности течения постинфарктного периода 76 больных, госпитализированных в отделении интенсивной терапии «Городского кардиологического центра» (г.Алматы) и Кардиохирургическом центре Мангыстауской областной больницы (г. Мангыстау) с диагнозом трансмуральный инфаркт задней стенки миокарда, с учетом возможности реализации кардиальноэнтерального тормозного рефлекса. Диагноз инфаркт миокарда был подтвержден наличием выраженных изменений на электрокардиограммах. Все больные, рассматриваемые в этой работе, дали письменное информированное согласие на участие в исследовании.

В зависимости от применения целенаправленной профилактики и коррекции нарушений пищеварительного тракта больных разделили на 2 группы.

В I группу вошли 34 больных, которым в постинфарктном периоде профилактика нарушений функционального состояния пищеварительного тракта, обусловленных кардиально-энтеральным тормозным рефлексом, не проводилась.

Во II группе у 42 больных в постинфарктном периоде проводились мероприятия, направленные на нормализацию функционального состояния кишечника и коррекцию последствий реализации кардиальноэнтерального тормозного рефлекса. Для этого при необходимости проводили декомпрессию желудка, проведение перманентных блокад 
забрюшинных нервных сплетений и назначение форлакса по 1 стандартному пакету, разведенному на 150 мл воды 6 раз в сутки.

Возраст больных в исследуемых группах колебался от 36 до 69 лет. Средний возраст

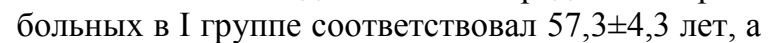
во II группе - 55,9 55,7 лет и достоверных различий между группами по возрасту выявлено не было ( $>0,05)$.

В I группе было $25(73,5 \pm 7,5 \%)$ мужчин и 9 $(26,5 \pm 7,5 \%)$ женщин, а во II группе $30(71,4 \pm 7,0 \%)$ мужчин и $12(28,6 \pm 7,0 \%)$ женщин.
Соответственно, достоверных различий между исследуемыми группами по половому составу не отмечалось ( $>>0,05)$.

В течение первых 12 часов после начала заболевания были госпитализированы более 80,0\% больных как в I, так и во II группе (таблица 1). Вероятно, более позднее обращение остальных больных было связано с менее выраженной клинической картиной, что может наблюдаться при поражении задней стенки миокарда. При этом достоверных различий между группами выявлено не было (P> 0,05).

Время, прошедшее от начала заболевания до госпитализации.

Таблица 1

\begin{tabular}{|c|c|c|c|c|c|}
\hline \multirow{2}{*}{$\begin{array}{c}\text { Время от начала заболе-вания до } \\
\text { госпитализации }\end{array}$} & \multicolumn{2}{|c|}{ I группа (n=34) } & \multicolumn{2}{|c|}{ II группа (n=42) } & \multirow{2}{*}{$\mathrm{P}$} \\
\cline { 2 - 6 } & Абс & $\%$ & Абс & $\%$ & $\mathrm{p}>0,05$ \\
\hline до 6 часов & 12 & $35,3 \pm 8,1$ & 13 & $30,9 \pm 7,1$ & $\mathrm{p}>0,05$ \\
\hline 6-12 часов & 17 & $50,0 \pm 8,5$ & 22 & $52,4 \pm 7,7$ & $\mathrm{p}>0,05$ \\
\hline $12-24$ часа & 4 & $11,8 \pm 5,5$ & 5 & $11,9 \pm 5,0$ & $\mathrm{p}>0,05$ \\
\hline
\end{tabular}

$P$ - достоверность различий между группами

Таблица 2

Тяжесть состояния больных при поступлении.

\begin{tabular}{|c|c|c|c|c|c|}
\hline \multirow[t]{2}{*}{ Состояние больных } & \multicolumn{2}{|c|}{ I группа $(\mathrm{n}=34)$} & \multicolumn{2}{|c|}{ II группа (n=42) } & \multirow[b]{2}{*}{$\mathrm{P}$} \\
\hline & Абс & $\%$ & Абс & $\%$ & \\
\hline Удовлетворительное & - & - & - & - & - \\
\hline Средней тяжести & 21 & $61,8 \pm 8,3$ & 24 & $57,1 \pm 7,6$ & $\mathrm{p}>0,05$ \\
\hline Тяжелое & 13 & $38,2 \pm 8,3$ & 18 & $42,9 \pm 7,6$ & $\mathrm{p}>0,05$ \\
\hline
\end{tabular}

$P$ - достоверность различий между группами

При поступлении 13 (38,2 $\pm 8,3 \%)$ больных в I группе и $18 \quad(42,9 \pm 7,6 \%)$ во II были госпитализированы в отделение интенсивной терапии в тяжелом состоянии (таблица 2).

При сравнении исследуемых групп на наличие у больных сопутствующих заболеваний достоверных различий между группами выявлено не было $(\mathrm{p}>0,05)$.

Наиболее часто в исследуемых группах встречались заболевания органов мочевыделения (хронический пиелонефрит, цистит и др.), пищеварения (хронический гастрит, язвенная болезнь желудка, хронический холецистит) и кровообращения (атеросклероз, облитерирующий эндартериит, варикозное расширение вен нижних конечностей).
Патология со стороны органов дыхания и мочевыделения встречалась реже, а патология опорно-двигательного аппарата, нервной и эндокринной систем лишь в единичных случаях.

Таким образом, исследуемые группы соответствовали друг другу и были сопоставимы $(\mathrm{p}>0,05)$.

При поступлении всем больным II группы проводили измерение расстояния между пупком и между пупком и передними верхними остями подвздошных костей. Обоснованность подобных измерений была связана с тем, что топографическое расположение того или иного отдела кишечника привязывается к верхним или нижним квадрантам брюшной полости, что позволяло при их увеличении в постинфарктном периоде диагностировать локальные нарушения пассажа по пищеварительному тракту. 
В постинфарктном периоде показаниями для декомпрессии желудка было увеличение расстояния между пупком и мечевидным отростком, по сравнению с показателями, зарегистрированными при поступлении. Декомпрессию и промывание желудка производили через назогастральный зонд, который устанавливали по мере надобности.

В зависимости от стороны, где отмечалось увеличение расстояния между пупком и передними верхними остями подвздошных костей, под контролем ультразвукового исследования производили пункционную катетеризацию забрюшинных нервных сплетений. При локализации увеличения исследуемого расстояния справа катетер устанавливали между куполом слепой кишки и бифуркацией правой общей подвздошной артерии, после чего по катетеру вводят 80,0-100,0 мл $0,25 \%$ раствора новокаина 4 раза в сутки. Такой объем вводимо местного анестетика позволял производить блокаду зоны илеоцекального сплетения и параректальных нервных образований.

При локализации увеличения исследуемого расстояния слева катетер устанавливали в корень брыжейки сигмовидной кишки в зоне ректосигмоидного перехода, после чего по катетеру вводят 80,0-100,0 мл раствора местного анестетика. Введение такого количества анестетика обеспечивало как блокаду нервных образований брыжейки сигмовидной кишки, так и параректальных нервных образований.

В зависимости от локализации нарушения пассажа химуса на «Способы катетеризации и блокады забрюшинных нервных образований поданы 2 заявки о выдаче патентов на изобретения РФ.

Статистическая обработка материала проводилась на ЭВМ с использованием вариационной статистики, определением средней арифметической $(\mathrm{M})$, ошибки средней арифметической (m) и расчетом критерия достоверности различий (t) по формуле и таблице Стьюдента.

\section{Результаты исследования}

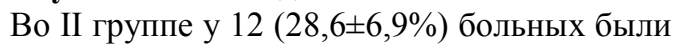
проведены декомпрессия и промывание желудка. Кроме того, по результатам антропометрических измерений, произведенных через сутки после поступления у $23(54,8 \pm 7,6 \%)$ произведена катетеризация забрюшинного пространства справа и у $19(45,2 \pm 7,6 \%)$ слева. При этом все больные II группы получали в постинфарктном периоде форлакс по 1 стандартному пакетику, разведенном в 200 мл воды 6 раз в сутки.

На фоне проведения перманентных блокад и других мероприятий, направленных на нормализацию функционального состояния кишечника (таблица 3), во II группе частота клинической симптоматики пареза кишечника выявлялась достоверно реже, чем в I $(\mathrm{P}<0,05)$.

\section{Таблица 3}

\section{Клиническая симптоматика нарушений функционального состояния кишечника в исследуемых} группах в постинфарктном периоде.

\begin{tabular}{|c|c|c|c|c|}
\hline \multirow[t]{2}{*}{ Клинические симптомы } & \multicolumn{2}{|c|}{ I группа (n=34) } & \multicolumn{2}{|c|}{ II группа $(\mathrm{n}=42)$} \\
\hline & Абс & $\%$ & Абс & $\%$ \\
\hline Нарушение дефекации & 24 & $70,6 \pm 8,9 *$ & 7 & $16,7 \pm 5,6^{*}$ \\
\hline Вздутие живота вне приема пищи & 15 & $44,1 \pm 8,5$ & 3 & $7,1 \pm 3,9 *$ \\
\hline Чувство тяжести в эпигастральной области после еды & 19 & $55,9 \pm 8,5^{*}$ & 5 & $11,9 \pm 5,0 *$ \\
\hline Вздутие живота (кишечника) сразу после еды & 17 & $50,0 \pm 8,5^{*}$ & 4 & $9,5 \pm 4,5^{*}$ \\
\hline $\begin{array}{l}\text { Вздутие живота (кишечника) через } 30-60 \text { минут после } \\
\text { еды }\end{array}$ & 26 & $76,5 \pm 7,3^{*}$ & 8 & $19,0 \pm 6,1^{*}$ \\
\hline Боли в животе на фоне вздутия, & 14 & $41,2 \pm 8,4^{*}$ & - & - \\
\hline Частота пареза кишечника & 26 & $76,5 \pm 7,3^{*}$ & 9 & $21,4 \pm 6,2 *$ \\
\hline Восстановление дефекации (сут) & \multicolumn{2}{|c|}{$5,6 \pm 0,4^{*}$} & \multicolumn{2}{|c|}{$2,8 \pm 0,5^{*}$} \\
\hline
\end{tabular}

* - достоверность различий между исследуемыми группами

Полученные, в результате иследований данные свидетельствовали о высокой клинической эффективности проводимых во II группе мероприятий по коррекции постинфарктных нарушений моторной и эвакуаторной функции кишечника. Это подтверждалось и иезультатами антропометрических измерений, проводимых в постинфарктном периоде (таблица 4). 
На фоне спазма сфинктера и нарушении пассажа из проксимальных отделов пищеварительного тракта в дистальные развивается стаз химуса, а на фоне брожения и гниения с повышенным газообразованием, и гипертензия в проксимальных отделах, что приводит к изменению антропометрических параметров передней брюшной стенки в зоне проекции соответствующего отдела кишечника.

Таблица 4

Частота изменения антропометрических параметров передней брюшной стенки через 3 суток после госпитализации.

\begin{tabular}{|l|c|c|c|c|}
\hline Исследуемые показатели & \multicolumn{2}{|c|}{ I группа (n=34) } & \multicolumn{2}{|c|}{ ІІ группа (n=42) } \\
\cline { 2 - 5 } & Абс & $\%$ & Абс & $2,4 \pm 2,3$ \\
\hline Ассиметрия передней брюшной стенки натощак & 8 & $23,5 \pm 7,2$ & 1 & $11,9 \pm 5,0 *$ \\
\hline Ассиметрия передней брюшной стенки после еды: & 25 & $73,5 \pm 7,5^{*}$ & 5 & $7,1 \pm 3,9 *$ \\
\hline $\begin{array}{l}\text { Увеличение расстояния между пупком и мечевидным } \\
\text { отростком }\end{array}$ & 15 & $44,1 \pm 8,5$ & 3 & $11,9 \pm 5,0 *$ \\
\hline $\begin{array}{l}\text { Увеличение расстояния между пупком и spina iliaca ant. } \\
\text { sup. dextra }\end{array}$ & 18 & $52,8 \pm 8,5 *$ & 5 & $7,1 \pm 3,9 *$ \\
\hline $\begin{array}{l}\text { Увеличение расстояния между пупком и spina iliaca ant. } \\
\text { sup. sinistra }\end{array}$ & 13 & $38,2 \pm 8,3$ & 3 & 7,3 \\
\hline
\end{tabular}

* - достоверность различий между исследуемьми группами

При сравнении антропометрических параметров передней брюшной стенки в постинфарктном периоде (таблица 4) было установлено, что показатели в I группе после еды, то есть характеризующие функциональное состояние пищеварительного тракта свидетельствовали о достоверно большей частоте нарушения пассажа как в зоне илеоцекального угла, так и по сигмовидной и дистальным отделам толстой кишки $(\mathrm{P}<0,05)$.

Следует отметить, что в I группе после еды более часто отмечалось и нарушение пассажа из желудка, но различия со II, были недостоверными $(\mathrm{P}<0,05)$.

\section{Обсуждение результатов исследования}

Сердце и пищеварительный тракт имеют общие источники иннерваци, что и определяет их рефлекторные взаимные влияния. При этом наиболее подвержены воздействию патологической импульсации зоны имеющие интенсивную иннервацию, в пищеварительном тракте это заны анатомических и функциональных сфинктеров.

Соответственно, именно в этих зонах кишечника, на фоне реализации кардиальноэнтерального тормозного рефлекса и будет происходить нарушение пассажа химуса даже на фоне восстановления перистальтики. То есть, кардиально-энтеральный тормозной рефлекс будет играть роль триггера развития нарушений моторной и эвакуаторной функции кишечника в постинфарктном периоде.

Патологическая импульсация кардиальноэнтерального тормозного рефлекса за счет нарушения функции запирательного аппарата, регламентирующего дозированное поступление химуса из проксимальных отделов в дистальные будет создавать предпосылки для его стаза, брожения и гниения. Следствием этого будет повышенное газообразованиеи развитие внутрипросветной гипертензии, которая за счет раздражения барорецепторов опосредованно приводит к усугублению спазма сфинктеров.

Наиболее уязвимыми в этом отношении являются анатомические сфинктеры привратник, илеоцекальный угол и анальный сфинктер, а также анатомический -зона сигмовидной кишки и ректосигмоидного перехода.

При нарушении пассажа из желудка, его декомпрессию проводит через зонд, который по мере надобности устанавливают в его просвет.

При нарушении дефекации можно сделать очистительную клизму, либо поставить газоотводную трубку.

Однако раздражение анального сфинктера приводит к развитию анально-энтерального тормозного рефлекса, следствием чего является спазм илеоцекального сфинктера и снижение перистальтики кишечника, в первую очередь его дистальных отделов.

Проведение таких сочетанных мероприятий, a, именно, забрюшинных блокад, рбеспечивающих восстановление пассажа по кишечнику и релаксацию анального сфинктера, по показаниям декомпрессии желудка в сочетании с пероральным приемом форлакса обеспечивает нормализацию функционального состояния пищеварительного тракта. 


\begin{tabular}{l|lrl|l|ll} 
& ISRA (India) & $=\mathbf{1 . 3 4 4}$ & SIS (USA) & $=\mathbf{0 . 9 1 2}$ & ICV (Poland) & $=\mathbf{6 . 6 3 0}$ \\
Impact Factor: & ISI (Dubai, UAE) $=\mathbf{0 . 8 2 9}$ & PUHL (Russia) $=\mathbf{0 . 1 7 9}$ & PIF (India) & $=\mathbf{1 . 9 4 0}$ \\
& GIF (Australia) & $\mathbf{0 . 5 6 4}$ & ESJI (KZ) & $=1.042$ & & \\
& JIF & $\mathbf{1 . 5 0 0}$ & SJIF (Morocco) $=\mathbf{2 . 0 3 1}$ & &
\end{tabular}

Полученные в клинике данные свидетельствовали о положительном влиянии перманентных блокад забрюшинных нервных образований при коррекции нарушений моторной и эвакуаторной функции кишечника, обусловленных кардиально-энтеральным тормозным рефлексом.

Особого внимания заслуживает и то, что, начиная с 3 суток после госпитализации во II группе $(35,7 \pm 7,3 \%)$, по сравнению с I $(87,5 \pm 5,7 \%)$ отмечалось достоверное снижение нарушений сердечного ритма, что свидетельствовало положительном влиянии профилактики и коррекции нарушений функционального состояния кишечника в постинфарктном периоде при инфаркте задней стенки миокарда и патогенетической обоснованности их проведения.

\section{References:}

1. Ruff CT (2011) The evolving epidemiology of acute coronary syndromes/ C.T. Ruff, E. Braunwald // Nature Reviews Cardiology 8, 140-147 (March 2011) doi:10.1038/nrcardio.2010.199

2. Drüeke TB (2010) Atherosclerosis in CKD: differences from the general population / T.B. Drüeke, Z.A. Massy // Nature Reviews Nephrology 6, 723-735 (December 2010) doi:10.1038/nrneph.2010.143

3. Camici PG (2014) Coronary microvascular dysfunction: mechanisms and functional assessment / P. G. Camici, G. d'Amati, O. Rimoldi // Nature Reviews Cardiology, 2014. 12, 48-62. doi:10.1038/nrcardio.2014.160. Published online 14 October 2014

4. Miura K, Nagai M, Ohkubo T (2013) Epidemiology of hypertension in Japan / $\mathrm{K}$. Miura, M. Nagai, T. Ohkubo // Circ J 2013; 77: 2226-2231.

5. Salekhov SA, Gordeev MN, Salekhova YS, Korabelnikova IA (2015) Influence of emotional and informational factors in implementation of coping strategies in psychological stress. ISJ Theoretical \& Applied Science 11 (31): 147-154. Soi: http://s-oi.org/1.1/TAS-11-31-24 Doi: http://dx.doi.org/10.15863/TAS.2015.11.31.24

6. Kenzhebaev AM (2011) Vliyanie ishemii peredney stenki miokarda na funktsionalnoe sostoyanie pischevaritelnogo trakta V eksperimente. / A.M. Kenzhebaev// Mat. Mezhdunarodnoy konf. «Zdorove i obrazovanie v XXI veke. - 2011. - t. 13. - pp.612-613

7. Minushkin ON (2002) Abdominalnaya bol: differentsialnaya diagnostika, vozmozhnyie lechebnyie podhodyi / O.N. Minushkin // Russkiy meditsinskiy zhurnal, 2002. - \#15. - p. 625.
8. Salehova MP, Shanin VY, Kudaybergenova RZ, Salehov SA (2004) Holetsisto-koronarnyiy i koronarno-holetsistitnyiy refleks pri ishemicheskoy bolezni serdtsa i holetsistite: vozmozhnosti differentsialnoy diagnostiki. // Klinicheskaya patofiziologiya. - 2004. - \#2. pp. 76-79.

9. Veber VR (2015) Patogeneticheskie osobennosti razvitiya vnutriprosvetnoy gipertenzii $\mathrm{v}$ kishechnike na fone eksperimentalnogo kardialno-enteralnogo refleksa / V.R. Veber, E.A. Adilbekov, M.P. Salehova i soavt. // Vestnik KRSU. 2015. - t. 15, \# 11. - pp. 42-45.

10. Salehov SA (2014) Sposob differentsialnoy diagnostiki kardiogennoy ishemii miokarda $\mathrm{i}$ enteralno-kardialnogo tormoznogo refleksa / S.A. Salehov, V.R. Veber, M.P. Salehova, A.I. Korabelnikov, E.A. Adilbekov // Patent na izobretenie RF \# 2535621. Zaregistrirovan v Gosudarstvennom reestre izobreteniy Rossiyskoy Federatsii 15.10.2014.

11. Korabelnikov AI (2015) Sposob differentsialnoy diagnostiki kardiogennoy ishemii miokarda i ileotsekalno-kardialnogo tormoznogo refleksa / A.I. Korabelnikov, V.R. Veber, M.P. Salehova i soavt // Patent na izobretenie RF \# 2541825. Opublikovan 2.02.2015, Byul. \# 5 .

12. Salehov SA (2014) Sposob differentsialnoy diagnostiki kardiogennoy ishemii miokarda i anorektalno-kardialnogo tormoznogo refleksa / S.A. Salehov, A.I. Korabelnikov, V.R. Veber, M.P. Salehova, E.A. Adilbekov // Patent na izobretenie RF \# 2535623. Zaregistrirovan v Gosudarstvennom reestre izobreteniy Rossiyskoy Federatsii 15.10.2014. 